\title{
The Effect of Photoluminescence of Bioceramic Irradiation on Middle Cerebral Arterial Occlusion in Rats
}

\author{
Lei Zhang, ${ }^{1}$ Paul Chan, ${ }^{2}$ Zhong-Min Liu, ${ }^{3}$ Ling-Ling Hwang, ${ }^{4}$ Kuo-Chi Lin, ${ }^{5}$ Wing P. Chan, \\ Ting-Kai Leung, ${ }^{6,7,8}$ and Cheuk Sing Choy \\ ${ }^{1}$ Department of Radiology, Shanghai East Hospital, Tongji University, 1800 Yantai Road, Shanghai 200123, China \\ ${ }^{2}$ Division of Cardiology, Department of Internal Medicine, Wan Fang Hospital, Taipei Medical University, \\ No. 111, Sec. 3, Singlong Road, Taipei 116, Taiwan \\ ${ }^{3}$ Department of Cardiac Surgery, Shanghai East Hospital, Tongji University, 1800 Yantai Road, Shanghai 200123, China \\ ${ }^{4}$ Department of Physiology, Taipei Medical University, No. 250, Wu Hsing Street, Taipei 110, Taiwan \\ ${ }^{5}$ Department of Diagnostic Radiology, Cathay General Hospital, 280 Renai Road, Sec. 4, Taipei 106, Taiwan \\ ${ }^{6}$ Department of Radiology, Wan Fang Hospital, Taipei Medical University, No. 111, Sec. 3, Singlong Road, Taipei 116, Taiwan \\ ${ }^{7}$ Graduate Institute of Biomedical Materials and Tissue Engineering, College of Medical Engineering, Taipei Medical University, \\ No. 250, Wu Hsing Street, Taipei 110, Taiwan \\ ${ }^{8}$ Department of Radiology, Taipei Hospital, Ministry of Health and Welfare, No. 127 Su Yuan Road, Hsinchuang District, \\ New Taipei City 242-13, Taiwan \\ ${ }^{9}$ Department of Emergency, Min-Sheng General Hospital, 168 Ching-Kuo Road, Taoyuan 330, Taiwan
}

Correspondence should be addressed to Ting-Kai Leung; hk8648@tmu.edu.tw

Received 20 January 2016; Revised 12 April 2016; Accepted 27 April 2016

Academic Editor: Menaka C. Thounaojam

Copyright (C) 2016 Lei Zhang et al. This is an open access article distributed under the Creative Commons Attribution License, which permits unrestricted use, distribution, and reproduction in any medium, provided the original work is properly cited.

\begin{abstract}
The purpose of this study is to determine the possible effect of photoluminescence of bioceramic (PLB) on ischemic cerebral infarction (stroke), by using an animal model of transient middle cerebral artery occlusion (MCAO). Sprague-Dawley rats were used to induce MCAO to block the origin of the left MCAO; three months later, the positive chronic stroke rats were selected by running tunnel maze; the MCAO rats with significant chronic stroke and neurological defects were used for treadmill experiments with varying speed settings to test their capability for restoration after muscular fatigue under conditions of with and without PLB irradiation. As a result, PLB irradiation could improve exercise completion rate and average running speed during slow and fast treadmill settings. After PLB irradiation, the selected MCAO rats successfully completed all the second-round treadmill exercises at the maximum speed setting, and they had better restoration from muscular fatigue. An in vitro cell study on astrocytes of rats by bioceramic irradiation further demonstrated increased intracellular nitric oxide. To explain these results, we suggest that cortical brain stimulation of microcirculation and enhancement of peripheral muscular activity are the main causes of the improved exercise performance in MCAO rats by PLB.
\end{abstract}

\section{Introduction}

Ischemic stroke is the result of reduction in blood flow to the affected brain regions. It causes sudden loss of neurological function after hypoxia of brain tissue and then activates pathogenic cascades after ischemia, disrupting cell metabolism, which eventually results in brain cell death. Ischemic stroke is the biggest cause of physical disability in both developed and developing countries $[1,2]$. Different treatments for postischemic stroke to restore normal functioning come at a cost for public healthcare. Basically, the majority of cases with brain damage are not reversible, and recovery of motor function following stroke is limited. It was believed that a possible improvement could happen by functional compensation from residual neural tissue. Providing more effective therapeutic methods and restoring normal function to poststroke patients are still popular research topics in many countries. 
Our previous studies showed that bioceramic materials treatment can promote microcirculation [3] and upregulate calcium-dependent nitric oxide and calmodulin $[4,5]$. We previously demonstrated that bioceramic materials treatment promotes nitric oxide enhancement through calciumdependent nitric oxide syntheses [4,5]. Additionally, bioceramic materials treatment had demonstrated antioxidant effects by increasing hydrogen peroxide scavenging processes in RAW264.7 murine macrophages [6,7], MC3T3-E1 murine calvaria-derived osteoblast-like cells [8], and C2C12 murine myoblasts [9]. There are beneficial effects of bioceramic irradiation treatment on exercise performance, achieved by decreasing the amount of lactate dehydrogenase released, decreasing metabolic acid accumulation, decreasing tiredness, reducing skin temperature, and stabilizing respiration and heart rates during exercise $[9,10]$. The findings were also further proved by in vivo studies on antioxidative stress effects on myoblast cells and preventing fatigue on amphibian skeletal muscles during continuous stimulation $[9,10]$.

Bioceramic irradiation treatment has also been shown to activate the parasympathetic nervous system, which may improve the recovery of resting cardiac and respiratory rates following submaximal exercise [11]. Additionally, it also contributes to other biological, chemical, and physical properties and has other clinical applications [12-20].

Recently, we presented a series of physical-biological experiments on the material based on the characteristics of biological effects of electromagnetic nonionizing radiation [21], nonlinear photonic crystal [22], and photoluminescence $[23,24]$. The concept of the "photoluminescence effect" is combining the effects of bioceramic materials, a kind of photonic crystal that emits high-performance ranges of infrared rays, and the visible light spectrum (Figure 1). The characteristics of bioceramic materials may belong to the fifth force field, which belongs to a new concept of physics different from the other four force fields [25]. We attempt to show the possible beneficial effects of PLB on rehabilitation in postinfarcted rats, selected and assessed by their maze performance. Since maze experiments and treadmill exercise are common methods in evaluating postinfarcted rats' performance [26, 27], we adopted these methods to select rats with significant cerebral injuries and to assess the possible recovery of cerebral function under PLB treatment. We also studied the PLB effect by using carotid Doppler ultrasound measurement. The purpose of this study was to evaluate the effects of PLB on stroke, using animal studies after postischemic infarction.

\section{Materials and Methods}

The ceramic powder was obtained from the Laboratory of Radiology in Taipei Hospital (New Taipei City, Taiwan). The bioceramic material consisted of microsized particles produced primarily from different elemental components.

2.1. Photoluminescence of Bioceramic Materials (PLB). The concept and procedure of PLB irradiation involve the application of specially manufactured bioceramic materials that

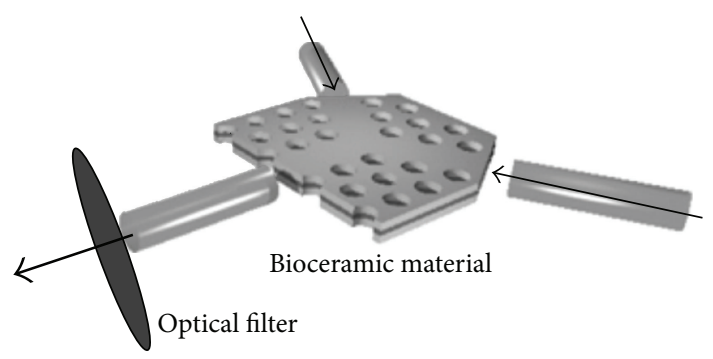

FIGURE 1: Conceptual picture of PLB, a combination of bioceramic material and visible light spectrum.

consists of microsized particles produced from various elemental components [4-20]. Of the bioceramic material, seven percent was embedded in a silicon sticker with good translucence (Bioenergy Development Ltd., Taoyuan, Taiwan). Photoluminescence is a special type of luminescence, referring to materials that absorb light energy and then release that energy in the form of light; it also describes the interaction between electromagnetic (EM) radiation and matter. The PLB of this bioceramic material absorbs a portion of the wavelength of the EM spectrum that includes near-, middle, and far infrared rays. The concept of PLB is a visible light source of light-emitting diodes (LEDs) without additional thermal effect, propagated through bioceramic materials for irradiation on living animals (Figure 1).

2.2. Animal Model Experiments. Sprague-Dawley rats (200 g; $n=10$ ) were maintained in cages at $23^{\circ} \mathrm{C} \pm 2^{\circ} \mathrm{C}$ and $60 \%$ $\pm 5 \%$ humidity with 12 -hour light/dark cycling. Solid rat food and water were continuously available during the first week to allow the rats to adjust to the environment. All procedures were conducted in accordance with the ethical guidelines for animal experiments established by our institution (IACVC approval number: LAC-101-0093). Anesthesia was induced with chloral hydrate dissolved in normal saline (35 mg/100 gm, intraperitoneal) and was maintained during the procedure by supplemental doses as needed. Body temperature was monitored with a rectal thermometer and was maintained within normal limits with a heating pad.

Transient MCAO was induced by advancing a 4-0 surgical nylon suture into the internal carotid artery (ICA) to block the origin of the MCA. A length of 18.5 to $19.5 \mathrm{~mm}$ 4-0 surgical nylon suture, determined by animal weight, was advanced from the left external carotid artery (ECA) into the lumen of the ICA until it blocked the origin of the MCA. The suture was withdrawn to restore blood flow and reperfusion after two hours and then the wound was clipped. As mentioned, selected rats were irradiated using a light source of combining LED (wavelength of visible light, as determined by spectroscopy by the Taiwan Textile Research Institute) and a photonic crystal of bioceramic material. The light sources were kept $10 \mathrm{~cm}$ from the rats' bodies (Figure 2). We also controlled the light illumination measured by illuminometer, constantly at $450 \pm 50$ lux, and irradiation for four hours as one section. 


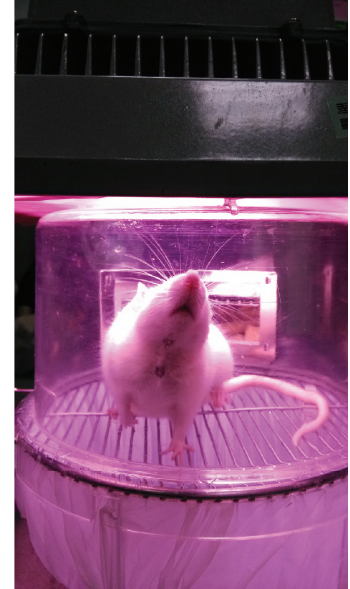

FIGURE 2: PLB irradiation on MCAO rats with surgical clips.

2.3. Assessment and Selection of MCAO Rats for PLB Treatment. To assess the amount of cerebral damage three months after the MCAO procedure (to be defined as chronic stroke status), we used a simple tunnel maze $(60 \times 45 \times 60 \mathrm{~cm})$, made of nontransparent material. Before a real test was performed, each rat received five practice runs in the tunnel maze, so as to develop experience and prevent fearfulness.

We selected the MCAO rats that expressed poor maze performance, those finishing the tunnel maze in over 200 seconds, to continue our tests.

2.4. Selected MCAO Rats for Tunnel Maze Experiment under PLB Treatment. The selected MCAO rats ran the tunnel maze without PLB and then received PLB treatment on another day at the same time, 2:00 p.m., so as to prevent uncontrollable factors on the levels of adrenaline and metabolic rates. The MCAO rats were kept in the tunnel maze for 200 seconds and 30-minute PLB treatments were then provided for each rat, and their subsequent tunnel maze performance was used for comparison.

2.5. Selected MCAO Rats for Treadmill Experiment under $P L B$ Irradiation. MCAO rats underwent the treadmill experiment at different speed settings of $10 \mathrm{~cm} / \mathrm{s}, 20 \mathrm{~cm} / \mathrm{s}, 30 \mathrm{~cm} / \mathrm{s}$, $40 \mathrm{~cm} / \mathrm{s}, 50 \mathrm{~cm} / \mathrm{s}, 60 \mathrm{~cm} / \mathrm{s}$, high speed $(80 \mathrm{~cm} / \mathrm{s})$, and maximum speed $(100 \mathrm{~cm} / \mathrm{s})$. From start to finish, the rats would complete three rounds of running at each speed setting. To be successful, the rat had to complete the entire distance; inability to complete the entire distance would be regarded as "failure" (Figure 3). Time required to finish the distance from the starting point to the finishing point was recorded for each MCAO rat. The distances of running speed per second were also calculated. We compared the control group (without PLB) and the experimental group (with PLB) to investigate whether the running velocity of MCAO rats could be improved.

2.6. PLB Irradiation on Selected MCAO Rats to Assess the Ability to Restore Muscular Fatigue. In the beginning, the selected MCAO rats finished the treadmill running and rested for 30 minutes without PLB irradiation. They were then divided into control and experimental (PLB irradiation) groups. The second round of treadmill running was designed with different treadmill speed settings to assess whether PLB irradiation could restore muscle fatigue on the selected MCAO rats.

\subsection{Selected MCAO Rats for Carotid Doppler Ultrasound} Experiment under PLB Irradiation. In this experiment, a Doppler ultrasound blood flow meter (BV 520, LG Biotech, Shanghai, China) was used to measure the MCAO rat carotid artery blood flow, including mean arterial blood flow velocity measurements $(\mathrm{MN})$ and the maximum spectral peak $(\mathrm{PK})$. First, the selected MCAO rats' hair was shaved from the dorsal neck, and then the Doppler ultrasound on carotid blood flow (DUCBF) was measured and recorded for PK and MN (Figure 4). For each selected MCAO rat, the DUCBF was measured four times: (1) before treadmill exercise; (2) after treadmill exercise; (3) after 30 minutes of rest with PLB irradiation before the second round of treadmill exercise; and (4) shortly after the second round of treadmill exercise.

\subsection{Measurement of Nitric Oxide (NO) Production on Primary} Cell Culture of Astrocyte with and without PLB Irradiation. Primary cultures of rat cortical neurons (provided by Professor Yi Hsuan Lee of the Department of Physiology, Taipei Medical University, Taiwan) were prepared from the cerebral cortex of 14-day-old rat foetuses. The cerebral cortices of foetuses obtained under sterile conditions were dissected and dissociated mechanically, by pipetting 10 times with $10 \mathrm{~mL}$ of DMEM (Gibco Invitrogen Corporation, Barcelona, Spain). The cell suspension was filtered through nylon mesh with a pore size of $90 \mu \mathrm{m}$. Cell suspension was plated $(5 \times$ $10^{4}$ cells $/ \mathrm{cm}^{2}$ ) on polylysine coated dishes. After attachment of the cells, the plating medium was changed to DMEM containing $10 \%$ FBS supplemented with antibiotics (1\%) and fungizone $(1 \%)$. Cultures were grown in a humidified atmosphere of $5 \% \mathrm{CO}_{2} / 95 \%$ air at $37^{\circ} \mathrm{C}$ for 3 days. Cells were then exposed to $10 \mu \mathrm{M}$ cytosine $\beta$-D-arabinofuranoside on the third day of culture for $24 \mathrm{~h}$ to inhibit proliferation of nonneuronal cells. The medium was changed twice a week.

The cells are separated as control and bioceramic irradiation groups, while the bioceramic irradiation group received bioceramic irradiation for 10 minutes. The cells and source of the bioceramic irradiation were separated by plastic culture discs, without direct contact (Figure 5), similar to MACO rats' brain tissue indirectly exposed from PLB.

All cell dishes were then stained with DAF-FM diacetate for fluorescence measurements for NO. All cells were analyzed by a fluorescence-activated cell sorter (FACS) and flow cytometry at the single-cell level. All the data were acquired and analyzed, and the mean fluorescence intensities of intracellular NO production in astrocytes of rats were determined.

2.9. Data Analysis. All experiments were done at least three times, each time with two or more independent observations. Statistical analysis was performed by paired $t$-test. 


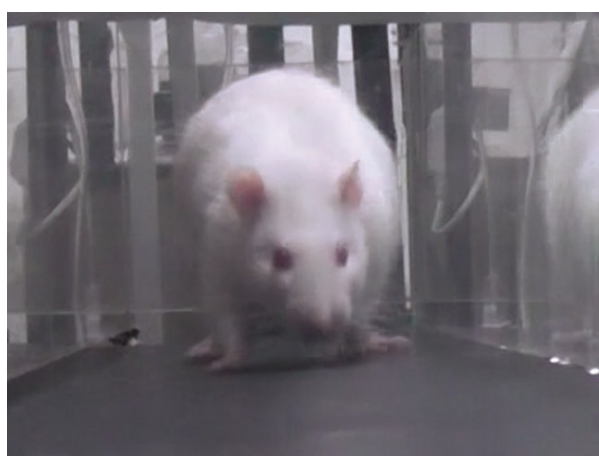

(a)

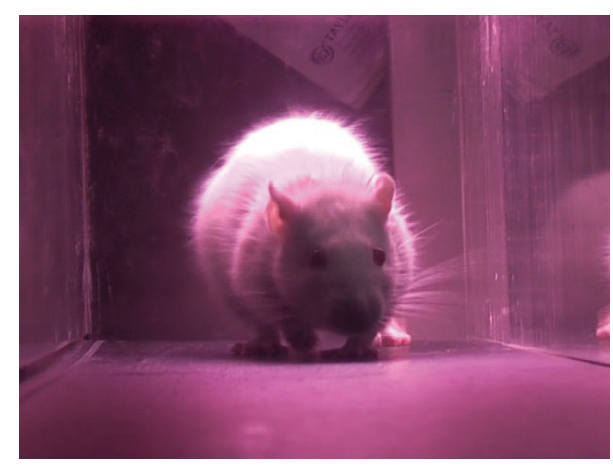

(b)

FIGURE 3: Running rat without PLB irradiation (a) and with PLB irradiation (b).

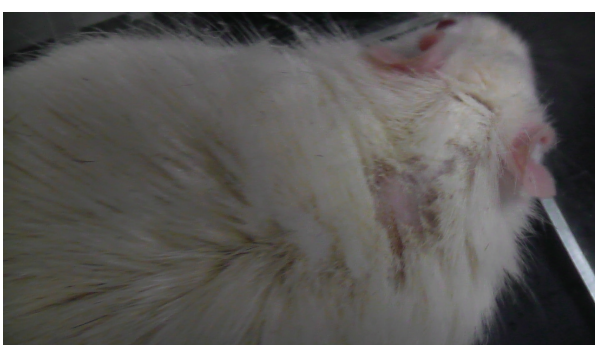

(a)

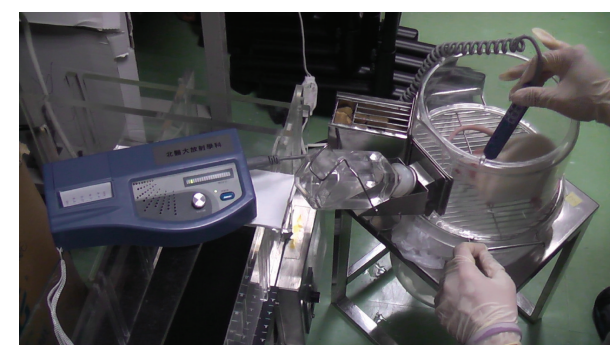

(b)

FIGURE 4: Hair shaved rats on the dorsal neck; (a) Doppler ultrasound measurement performed on carotid blood flow (b).

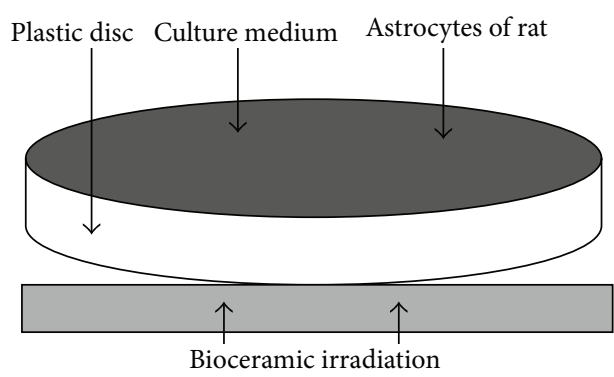

FIGURE 5: In vivo experimental model of bioceramic irradiation on astrocytes of rats, in addition to NO concentration measurement.

\section{Results}

3.1. Number of MCAO Rats Selected for PLB Treatment. After the MCAO procedure on ten rats, three of the rats expired within 24 hours of the surgery. Although the surviving rats initially expressed transient neurological deficits on motor behavior, all of the MCAO rats were nearly recovered in general appearance. In order to select the brain damaged rats after the MCAO procedure, the rats underwent tunnel maze tests. Three out of the seven rats exhibited poor performance on the tunnel maze tests, taking more than 200 seconds, and were selected for PLB treatments (Figure 6). This indicates that the selected MCAO rats had confirmed significant neurological defects.

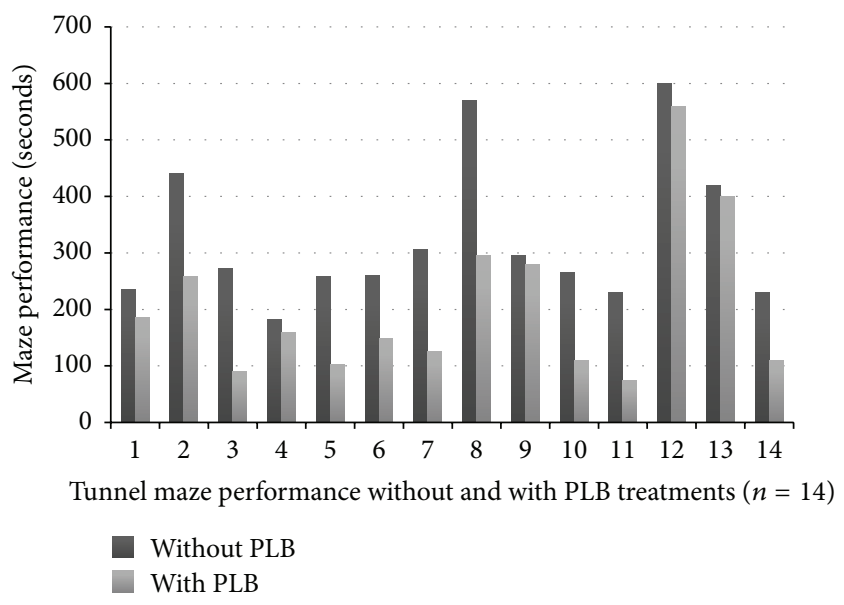

FIGURE 6: Comparison of MCAO rats on tunnel maze performance, with and without PLB.

3.2. PLB Treatment Reduces the Time Period Needed to Complete the Tunnel Maze in Selected MCAO Rats. Our data showed improvements in the required time for the three selected MCAO rats (number 3, number 4, and number 8) to complete the tunnel maze after PLB treatments $(n=14)$, which is a statistically significant difference $(p<0.05)$. Once the PLB treatment was provided for the selected MCAO rats, it was necessary to wait for two to three days until the disappearance of previous bioceramic irradiation effects. 


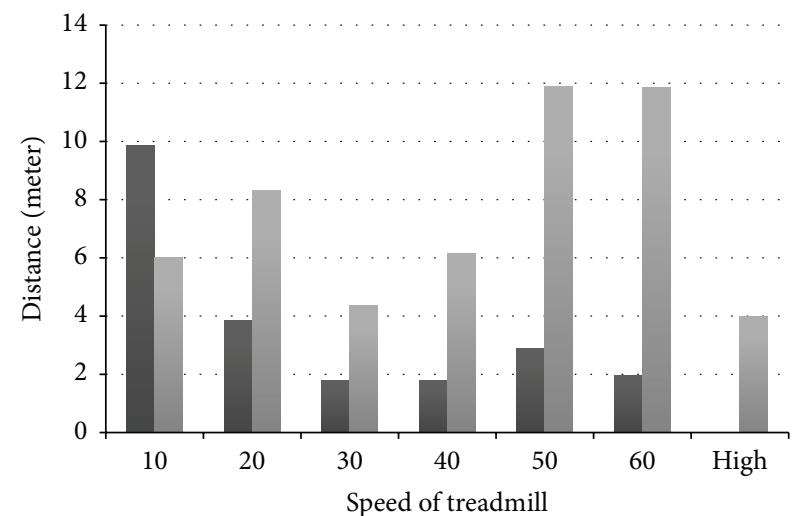

Without PLB With PLB

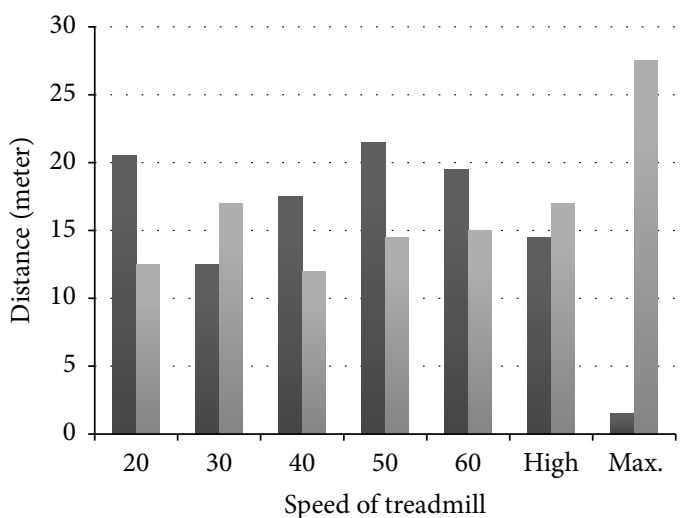

Without PLB

With PLB

(a)

(b)

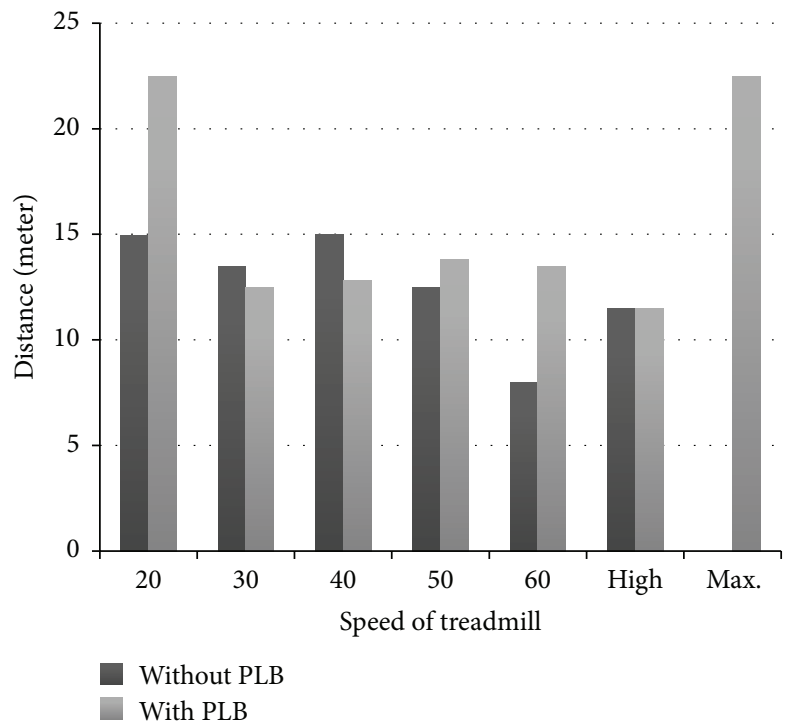

(c)

FIGURE 7: (a) The performance of number 3 rat with and without PLB, during running distance (meter) on treadmill under different speeds $(\mathrm{cm} / \mathrm{sec})$. (b) The performance of number 4 rat with and without PLB, during running distance (meter) on treadmill under different speeds $(\mathrm{cm} / \mathrm{sec})$. (c) The performance of number 8 rat with and without PLB, during running distance (meter) on treadmill under different speeds $(\mathrm{cm} / \mathrm{sec})$.

3.3. Results of PLB Treatment on Selected MCAO Rats for Treadmill Running. Based on statistical results of the selected MCAO rats from the second round of treadmill exercise to assess muscular fatigue restoration, it was found that PLB irradiation could enhance the completion rate and average speed (Figures $7(\mathrm{a})-7(\mathrm{c})$ ). For example, number 3 rat failed to finish an additional treadmill exercise without PLB irradiation and expressed exhaustion. It ran slowly and was then being dragged by the running treadmill at the end of the exercise. On the contrary, PLB irradiation promotes completion rate, faster running, and better achievements on all treadmill speed settings. Particularly, better performance after PLB irradiation on the second round of treadmill exercise was very significant on high-speed running. The average rate of running is $4.07 \pm 6.65 \mathrm{~cm} / \mathrm{s}$. The results of number 4 and number $8 \mathrm{MCAO}$ rats on the second round of treadmill exercises showed only mild improvement on their performance differences with PLB irradiation and without PLB irradiation. However, at maximum speed, there were significant differences; the average rate is $27.47 \pm 0.84$ for the PLB irradiation group and $22.54 \pm 19.91$ for the control group $(p<0.05)$.

3.4. Results of PLB Treatment on Selected MCAO Rats for Restoration of Muscular Fatigue. In the beginning, the MCAO rats failed to finish the exercise at the maximum speed setting. After PLB irradiation, it was found that number 3 , number 4 , and number $8 \mathrm{MCAO}$ rats completed all the second-round treadmill exercises at the maximum speed setting by an average speeds rate of $6.11 \pm 7.34 \mathrm{~cm} / \mathrm{s}$ (number 3 ), 


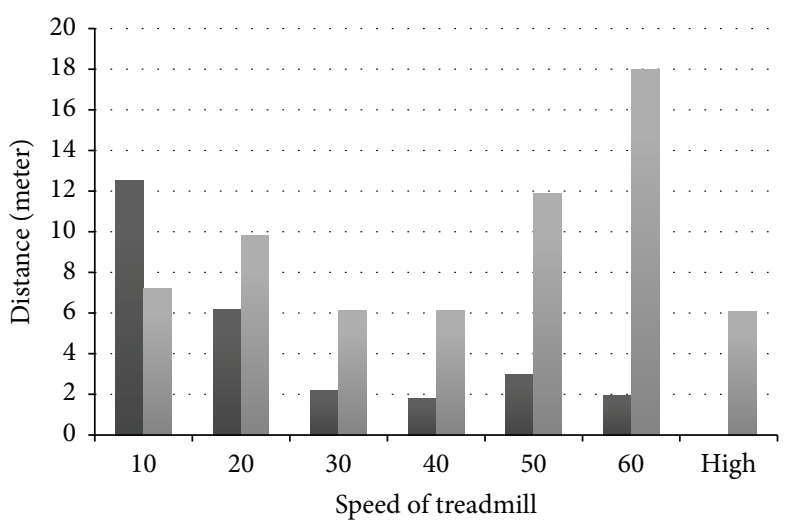

Without PLB With PLB

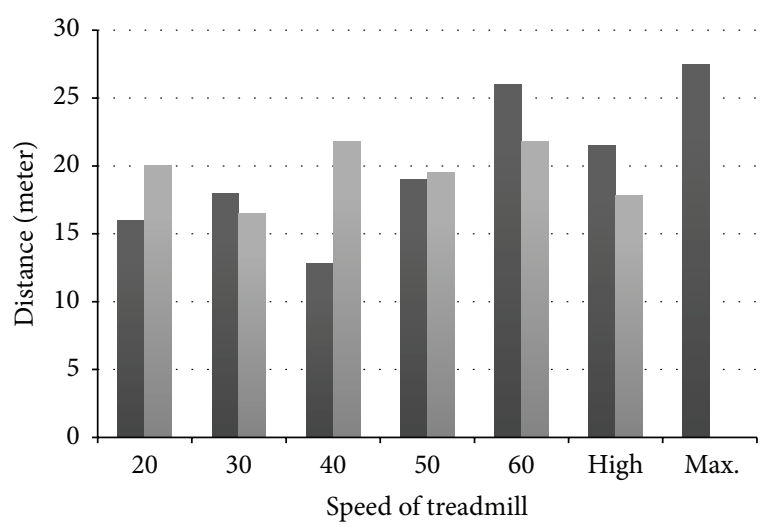

Without PLB

With PLB

(a)

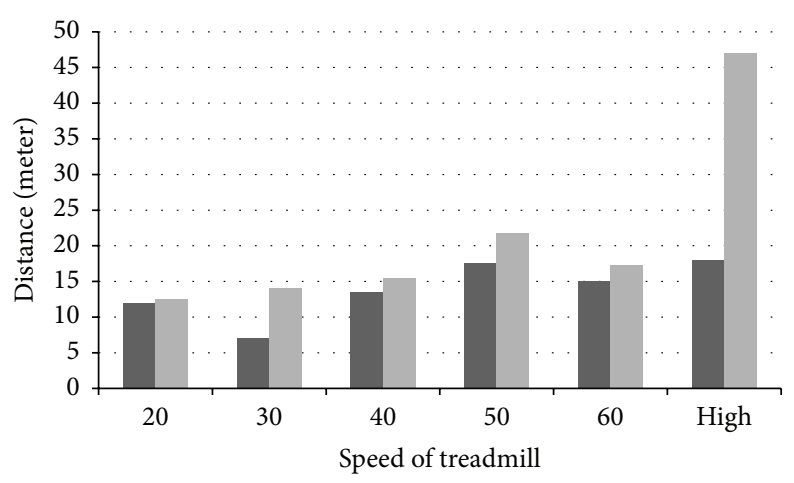

Without PLB

With PLB

(c)

Figure 8: (a) The performance of number 3 rat with and without PLB for restoration of muscular fatigue, by running distance (meter) on treadmill under different speeds $(\mathrm{cm} / \mathrm{sec})$. (b) The performance of number 4 rat with and without PLB for restoration of muscular fatigue, by running distance (meter) on treadmill under different speeds $(\mathrm{cm} / \mathrm{sec})$. (c) The performance of number 8 rat with and without PLB for restoration of muscular fatigue, by running distance (meter) on treadmill under different speeds $(\mathrm{cm} / \mathrm{sec})$.

$27.47 \pm 0.84 \mathrm{~cm} / \mathrm{s}$ (number 4 ), and $47 \pm 2.48 \mathrm{~cm} / \mathrm{s}$ (number 8) (Figures $8(\mathrm{a})-8(\mathrm{c}))$. Comparing the control and PLB irradiation groups shows a significant difference $(p<0.05)$.

\subsection{Results of PLB Treatment on Parameters of Carotid} Doppler Ultrasound. In the beginning, we predicted significant differences of MN and PK by DUCBF measurement of PLB irradiation before and after treadmill exercise. In comparing PLB irradiation before and after treadmill exercise, however, the results of DUCBF measurements showed no significant differences on PK and MN before and after PLB irradiation with $p>0.05$ (Table 1 ).

3.6. Results of Nitric Oxide Production on Astrocytes of Rats under PLB Irradiation. Levels of intracellular NO synthesis of astrocytes of rats in the control and bioceramic irradiation groups are shown in Figure 9. The $p$ value between these two groups is less than 0.05 , which shows a significant difference.

\section{Discussion}

Stroke is a leading cause of adult motor disability, and the corresponding recovery of motor function from stroke is usually a slow and frustrating process [26]. From our results, both maze performance and treadmill exercise could be improved by using PLB irradiation. In our opinion, cortical brain stimulation of microcirculation and enhancement of peripheral muscular activity are the main causes for improved performance in MCAO rats undergoing PLB. Before we discuss the possible mechanisms of how PLB irradiation affects the behavior of MCAO rats, we should review clinical methods that may improve poststroke or brain cortex ischemic injury of humans and rats. For example, clinical uses of repetitive transcranial electrical and magnetic stimulations were developed to improve brain activity after ischemic stroke [27]. For magnetic stimulation, a pulsed magnetic field creates current flow in the brain and can temporarily excite or inhibit specific areas. Transcranial magnetic stimulation 
TABLE 1: Results of selected MCAO rats for carotid Doppler ultrasound experiment with and without PLB irradiation ( $p$ value $>0.05$; experimental data exhibit no significant difference).

\begin{tabular}{lcccc}
\hline Number of MCAO rat & Timing of DUCBF measurement & $\begin{array}{c}\text { DUCBF measurement } \\
\text { without PLB }\end{array}$ & $\begin{array}{c}\text { DUCBF } \\
\text { measurement } \\
\text { with PLB }\end{array}$ & $\begin{array}{c}\text { Data subtraction of } \\
\text { measurement without } \\
\text { PLB from that with } \\
\text { PLB }\end{array}$ \\
\hline \multirow{3}{*}{ Number 3 } & Preexercise PK & 0.378 & 0.284 & 0.094 \\
& Postexercise PK & 0.336 & 0.473 & -0.137 \\
& Preexercise MN & 0.176 & 0.162 & 0.014 \\
& Postexercise MN & 0.183 & 0.217 & -0.034 \\
\hline \multirow{3}{*}{ Number 4 } & Preexercise PK & 0.432 & 0.444 & -0.012 \\
& Postexercise PK & 0.84 & 0.835 & 0.005 \\
& Preexercise MN & 0.217 & 0.254 & -0.037 \\
Number 8 & Postexercise MN & 0.34 & 0.59 & -0.25 \\
& Preexercise PK & 0.434 & 0.738 & -0.304 \\
& Postexercise PK & 1.163 & 0.7 & 0.463 \\
\hline
\end{tabular}

MN: mean arterial blood flow velocity measurements; PK: maximum spectral peak.

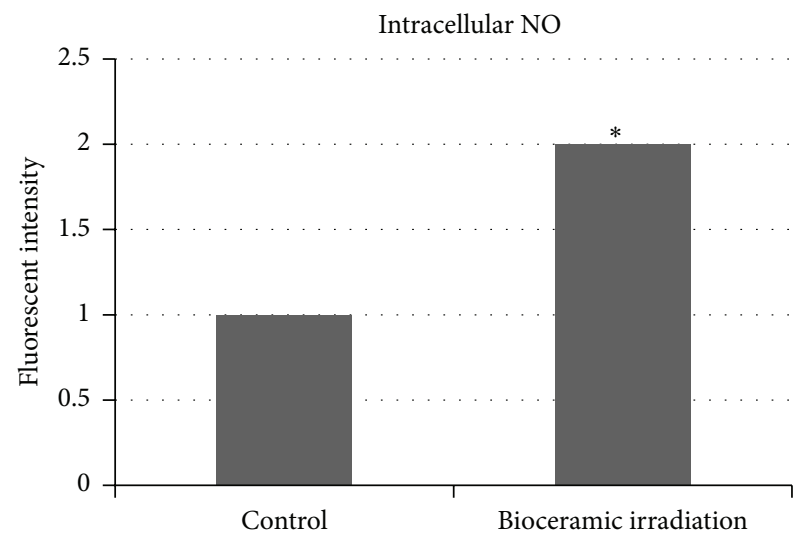

FIGURE 9: By cytometric analysis, NO production in bioceramic irradiation group within astrocytes of rats is statistically significantly increased $\left({ }^{*} p<0.05\right)$ compared with the control group.

of the motor cortex can produce muscle twitch or block movement; transcranial magnetic stimulation of occipital cortex can alter the brain function beyond the time of stimulation, offering potential for therapy. Electrical and magnetic methods are available to stimulate the human brain through the intact scalp. The effects of these less invasive cortical stimulations on the extended time course of learning have beneficial and promising implications on motor learning protocols in healthy individuals and in stroke patients undergoing neurorehabilitation $[28,29]$. However, the mechanisms of these two methods are not well understood and not widely used clinically. On the other hand, hyperbaric oxygen (HBO) therapy was also proven to have the capability to reduce ischemic brain damage and behavioral dysfunctions, which may reduce the infarct area and improve neurologic scores at seven days after reperfusion [28-31]. One of the molecular mechanisms of HBO-induced brain protection is preventing apoptosis, and this effect of $\mathrm{HBO}$ might preserve more brain tissues and promote neurologic functional recovery. HBO can reduce brain tissue apoptosis, especially when applied after the initial ischemia; HBO might be used as an alternative or additional therapy for patients who have had an acute stroke because no effective treatment is available [30,31]. The effects of reduced behavioral dysfunctions of ischemic stroke for both animals and patients by HBO therapy are related to upregulating nitric oxide that promotes microcirculation and helps recover damaged brain tissue, probably in acute and subacute stages [32-35]. However, there is still not enough quantitative measurement to determine the effectiveness of HBO treatment on recovery of chronic stroke patients [32].

In this study, PLB irradiation externally on chronic stroke rats is a new kind of cortical stimulation, without applying magnetism and strong electricity. We had previously demonstrated that bioceramic materials treatment was able to promote microcirculation in patients [3] by upregulating calcium-dependent nitric oxide and calmodulin in cell lines $[4,5]$. In this present study, we demonstrate an in vitro study on astrocytes of rats, with a statistically significant increase of NO production by bioceramic irradiation. Increasing the intracranial microcirculation by bioceramic irradiation is a kind of brain stimulation, acting as one of the reasons to explain how PLB improved exercise performance of MCAO rats on both maze and treadmill tests. Apart from brain stimulation, practice of rehabilitation on stroke patients is also achieved by strengthening peripheral muscles. Clinically, postinfarcted patients are trained for different walking speeds and inclinations on the treadmill platform, to provide treatment on different paretic and nonparetic sides of muscles [36]. For example, methods to improve medial gastrocnemius activity of poststroke patients may improve their treadmill performance [36]. One of our previous studies showed 
the effects of bioceramic materials on oxidative stressed murine myoblast cells $(\mathrm{C} 2 \mathrm{C} 12)$ and fatigue conditions of amphibian skeletal muscle during exercise [9]; this helped to explain PLB treatment on MCAO rats for restoration of muscular fatigue. We had demonstrated that bioceramic irradiation received beneficial effects on viability of $\mathrm{H}_{2} \mathrm{O}_{2}$ mediated oxidative stress induced $\mathrm{C} 2 \mathrm{C} 12$ cells and decreasing lactate dehydrogenase release and resulted with reduction of muscular fatigue [9]. It was proven that nitric oxide (NO) within $\mathrm{C} 2 \mathrm{C} 12$ cell was elevated after bioceramic irradiation. We had further used a physiological experimental model of electrostimulation on amphibian skeletal muscle, stimulating the muscle samples by continuous pulses for a long period of time and keeping record of contraction loading force until onset of muscle fatigue. In comparing the mean contraction force between the bioceramic irradiation group and control group, there was a significant difference $(p<0.05)$ between the two groups on the mean contraction loading forces, which indicates that bioceramic irradiation successfully reduced fatigue in amphibian muscles. Furthermore, measurement of $\mathrm{pH}$ changes on muscles after fatigue was done after the electrostimulated isometric contractions associated with bioceramic irradiation. The results showed a decrease on metabolic acid accumulation with measurable elevation of $\mathrm{pH}$ value on the muscular tissue, as compared to the control group. Thus, bioceramic irradiation can normalize acidification following muscle contractions and delay onset of muscular fatigue [9]. Additionally, a human trial of strenuous exercise in a model of treadmill running was performed with and without bioceramic irradiation, recorded with tendencies of decreased tiredness and reduced skin temperature in the bioceramic irradiation group. Moreover, more stable respiration rate and heart rate on candidates were recorded in the bioceramic irradiation group compared with the control group [10]. One of the limitations in this study is that the Doppler ultrasound measurement on MCAO rats of postPLB irradiation was not able to determine its effectiveness on $\mathrm{PK}$ and $\mathrm{MN}$. The reason is the technical difficulties in data collection on uncontrollable and moving rats. In the future, additional experiments or new methods will be required to approach PLB effect on carotid blood flow.

\section{Conclusion}

We found that PLB irradiation was able to improve different running speeds of the selected MCAO rats, particularly in maximum speed treadmill running and second-round exercise. We suggest that cortical brain stimulation of microcirculation and enhancement of peripheral muscular activity are the two main causes for better exercise performance in MCAO rats by PLB. In the future, medical instrument development for clinical PLB trials is necessary for chronic cerebral infarction. The above findings can be of important reference for future medical device development. Further research of human trial on the correlations among regional cerebral flow, neuromuscular performance, and motor restoration is necessary.

\section{Competing Interests}

The authors declare that they have no competing interests.

\section{Authors' Contributions}

Lei Zhang and Paul Chan contributed equally to this study.

\section{Acknowledgments}

This study was funded by Shanghai Science and Technology Committee of China (Grant no. 15DZ0503500). The study was also assisted by Professor Yi Hsuan Lee.

\section{References}

[1] X. Tong, E. V. Kuklina, C. Gillespie, and M. G. George, "Medical complications among hospitalizations for ischemic stroke in the United states from 1998 to 2007," Stroke, vol. 41, no. 5, pp. 980986, 2010.

[2] Y. Ma, Y. Liu, H.-M. Fu et al., "Evaluation of admission characteristics, hospital length of stay and costs for cerebral infarction in a medium-sized city in China," European Journal of Neurology, vol. 17, no. 10, pp. 1270-1276, 2010.

[3] Y. S. Lin, M. Y. Lin, T. K. Leung et al., "Properties and biological effects of high performance ceramic powder emitting farinfrared irradiation," Instruments Today, vol. 28, no. 6, pp. $60-$ 66, 2007.

[4] T.-K. Leung, C.-M. Lee, M.-Y. Lin et al., "Far infrared ray irradiation induces intracellular generation of nitric oxide in breast cancer cells," Journal of Medical and Biological Engineering, vol. 29, no. 1, pp. 15-18, 2009.

[5] T.-K. Leung, Y.-S. Lin, Y.-C. Chen et al., "Immunomodulatory effects of far-infrared ray irradiation via increasing calmodulin and nitric oxide production in raw 264.7 macrophages," Biomedical Engineering: Applications, Basis and Communications, vol. 21, no. 5, pp. 317-323, 2009.

[6] T.-K. Leung, Y.-S. Lin, C.-M. Lee et al., "Direct and indirect effects of ceramic far infrared radiation on the hydrogen peroxide-scavenging capacity and on murine macrophages under oxidative stress," Journal of Medical and Biological Engineering, vol. 31, no. 5, pp. 345-351, 2011.

[7] T.-K. Leung, H.-F. Shang, D.-C. Chen et al., "Effects of far infrared rays on hydrogen peroxide-scavenging capacity," Biomedical Engineering-Applications, Basis and Communications, vol. 23, no. 2, pp. 99-105, 2011.

[8] T.-K. Leung, C.-H. Chen, C.-H. Lai et al., "Bone and joint protection ability of ceramic material with biological effects," The Chinese Journal of Physiology, vol. 55, no. 1, pp. 47-54, 2012.

[9] T.-K. Leung, C.-M. Lee, S.-Y. Tsai, Y.-C. Chen, and J.-S. Chao, "A pilot study of ceramic powder far-infrared ray irradiation (cFIR) on physiology: observation of cell cultures and amphibian skeletal muscle," The Chinese Journal of Physiology, vol. 54, no. 4, pp. 247-254, 2011.

[10] T. K. Leung, C. H. Kuo, C. M. Lee, N. W. Kan, and C. W. Hou, "Physiological effects of BIOCERAMIC material on human, assessment by 'Harvard Step', 'Resting Metabolic Rate' (RMR) and 'Treadmill Running' tests,' Chinese Journal of Physiology, vol. 56, no. 6, pp. 334-340, 2013.

[11] T.-K. Leung, C.-H. Chen, S.-Y. Tsai, G. Hsiao, and C.-M. Lee, "Effects of far infrared rays irradiated from ceramic material 
(BIOCERAMIC) on psychological stress-conditioned elevated heart rate, blood pressure, and oxidative stress-suppressed cardiac contractility," The Chinese Journal of Physiology, vol. 55, no. 5, pp. 323-330, 2012.

[12] T.-K. Leung, P.-J. Huang, Y.-C. Chen, and C.-M. Lee, "Physicalchemical test platform for room temperature, far-infrared ray emitting ceramic materials (cFIR)," Journal of the Chinese Chemical Society, vol. 58, no. 5, pp. 653-658, 2011.

[13] B. Y. Liau, T. K. Leung, M. C. Ou, C. K. Ho, A. Yang, and Y. S. Lin, "Inhibitory effects of far-infrared ray-emitting belts on primary dysmenorrhea," International Journal of Photoenergy, vol. 2012, Article ID 238468, 6 pages, 2012.

[14] T.-K. Leung, C.-F. Chan, P.-S. Lai, C.-H. Yang, C.-Y. Hsu, and Y.S. Lin, "Inhibitory effects of far-infrared irradiation generated by ceramic material on murine melanoma cell growth," International Journal of Photoenergy, vol. 2012, Article ID 646845, 8 pages, 2012.

[15] T.-K. Leung, C.-M. Lee, S.-L. Lin et al., "Protective effect of nonionizing radiation from ceramic far infrared (cFIR)-emitting material against oxidative stress on human breast epithelial cells," Journal of Medical and Biological Engineering, vol. 34, no. 1, pp. 69-75, 2014.

[16] T.-K. Leung, J.-C. Yang, and Y.-S. Lin, “The physical, chemical and biological effects by room temperature ceramic far-infrared ray emitting material irradiated water: a pilot study," Journal of the Chinese Chemical Society, vol. 59, no. 5, pp. 589-597, 2012.

[17] T. K. Leung, Y. C. Liu, C. H. Chen, H. N. Fang, K. C. Chen, and C. M. Lee, "In vitro cell study of the possible anti-inflammatory and pain relief mechanism of far-infrared ray emitting ceramic material," Journal of Medical and Biological Engineering, vol. 33, no. 2, pp. 179-184, 2013.

[18] T.-K. Leung, M.-T. Lin, C.-A. Chen, C.-Y. Hsu, S.-L. Lin, and C. W. Chen, "The analysis of normalized effects on meridian current level after the photoluminescent bioceramic treatment on acupuncture points," Journal of Alternative and Complementary Medicine, vol. 21, no. 8, pp. 472-479, 2015.

[19] T.-K. Leung, "In vitro and in vivo studies of the biological effects of bioceramic (a material of emitting high performance farinfrared ray) irradiation," Chinese Journal of Physiology, vol. 58, no. 3, pp. 147-155, 2015.

[20] C. W. Chen, C.-J. Tai, C.-S. Choy et al., "Wave-induced flow in meridians demonstrated using photoluminescent bioceramic material on acupuncture points," Evidence-Based Complementary and Alternative Medicine, vol. 2013, Article ID 739293, 11 pages, 2013.

[21] S. Kwee and P. Raskmark, "Changes in cell proliferation due to environmental non-ionizing radiation 1 . ELF electromagnetic fields," Bioelectrochemistry and Bioenergetics, vol. 36, no. 2, pp. 109-114, 1995.

[22] V. Berger, "Nonlinear photonic crystals," Physical Review Letters, vol. 81, no. 19, pp. 4136-4139, 1998.

[23] G. D. Gilliland, "Photoluminescence spectroscopy of crystalline semiconductors," Materials Science and Engineering R: Reports, vol. 18, no. 3-6, pp. 99-400, 1997.

[24] H. Yu, S. Lycett, C. Roberts, and R. Murray, "Time resolved study of self-assembled InAs quantum dots," Applied Physics Letters, vol. 69, no. 26, pp. 4087-4089, 1996.

[25] B. Bertotti and C. Sivaram, "Radiation of the 'fifth-force' field," Il Nuovo Cimento B, vol. 106, article 1299, 1991.

[26] F. Hummel, P. Celnik, P. Giraux et al., "Effects of non-invasive cortical stimulation on skilled motor function in chronic stroke," Brain, vol. 128, no. 3, pp. 490-499, 2005.
[27] M. Hallett, "Transcranial magnetic stimulation and the human brain," Nature, vol. 406, no. 6792, pp. 147-150, 2000.

[28] K. S. Utz, V. Dimova, K. Oppenländer, and G. Kerkhoff, "Electrified minds: transcranial direct current stimulation (tDCS) and Galvanic Vestibular Stimulation (GVS) as methods of non-invasive brain stimulation in neuropsychology-a review of current data and future implications," Neuropsychologia, vol. 48, no. 10, pp. 2789-2810, 2010.

[29] D. Liebetanz, R. Koch, S. Mayenfels, F. König, W. Paulus, and M. A. Nitsche, "Safety limits of cathodal transcranial direct current stimulation in rats," Clinical Neurophysiology, vol. 120, no. 6, pp. 1161-1167, 2009.

[30] C. F. Changa, K. C. Niub, B. J. Hofferc, Y. Wangc, and C. V. Borlonganc, "Hyperbaric oxygen therapy for treatment of postischemic stroke in adult rats," Experimental Neurology, vol. 166, no. 2, pp. 298-306, 2000.

[31] D. Yin, C. Zhou, I. Kusaka et al., "Inhibition of apoptosis by hyperbaric oxygen in a rat focal cerebral ischemic model," Journal of Cerebral Blood Flow and Metabolism, vol. 23, no. 7, pp. 855-864, 2003.

[32] S. Carson, M. McDonagh, B. Russman, and M. Helfand, "Hyperbaric oxygen therapy for stroke: a systematic review of the evidence," Clinical Rehabilitation, vol. 19, no. 8, pp. 819-833, 2005.

[33] F. M. Faraci and J. E. Brian Jr., "Nitric oxide and the cerebral circulation," Stroke, vol. 25, no. 3, pp. 692-703, 1994.

[34] C. F. Chang, K. C. Niu, B. J. Hoffer, Y. Wang, and C. V. Borlongan, "Hyperbaric oxygen therapy for treatment of postischemic stroke in adult rats," Experimental Neurology, vol. 166, no. 2, pp. 298-306, 2000.

[35] J. A. Buras, G. L. Stahl, K. K. H. Svoboda, and W. R. Reenstra, "Hyperbaric oxygen downregulates ICAM-1 expression induced by hypoxia and hypoglycemia: the role of NOS," American Journal of Physiology-Cell Physiology, vol. 278, no. 2, pp. C292-C302, 2000.

[36] R. Mohammadi, S. Talebian, C. P. Phadke, M. S. Yekaninejad, and M. Hadian, "Effects of treadmill incline and speed on ankle muscle activity in subjects after a stroke," Archives of Physical Medicine and Rehabilitation, vol. 97, no. 3, pp. 445-453, 2016. 


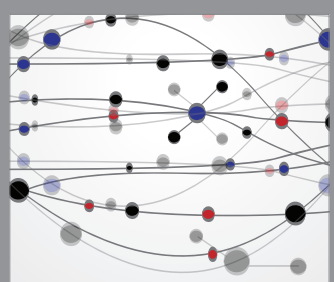

The Scientific World Journal
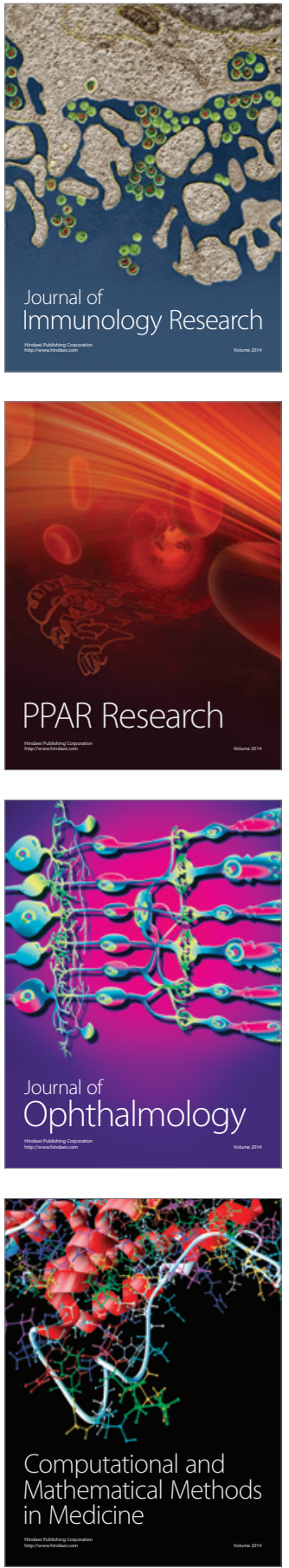

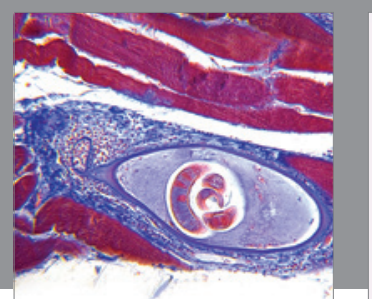

Gastroenterology Research and Practice

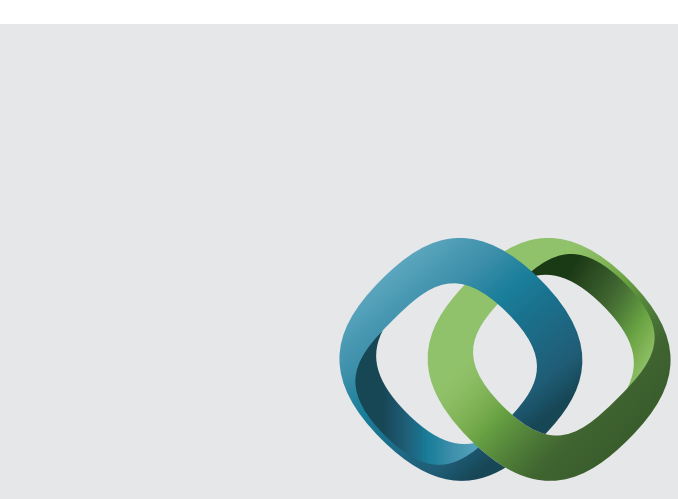

\section{Hindawi}

Submit your manuscripts at

http://www.hindawi.com
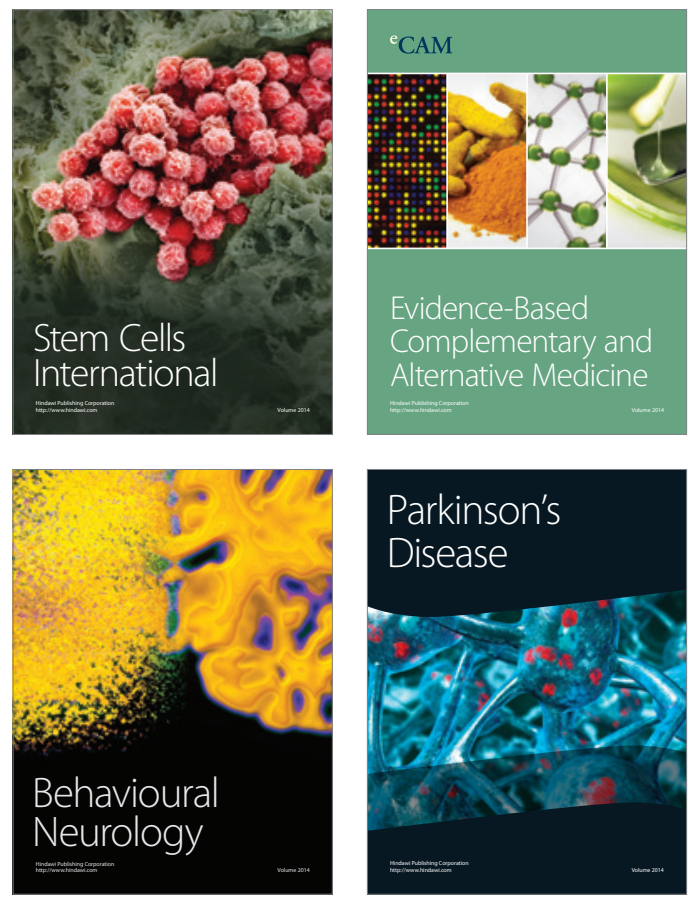
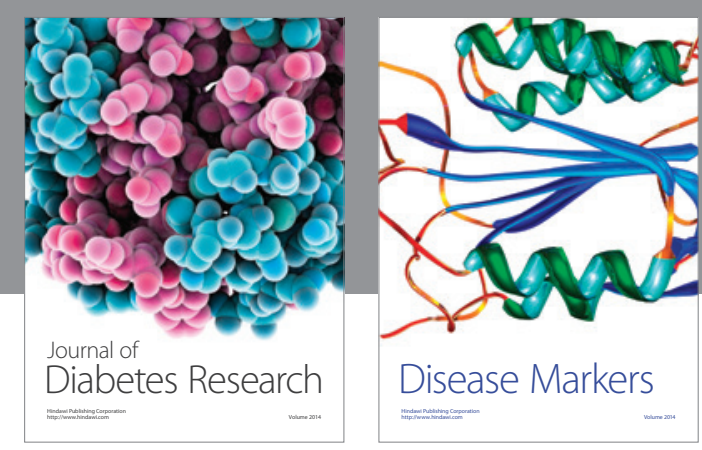

Disease Markers
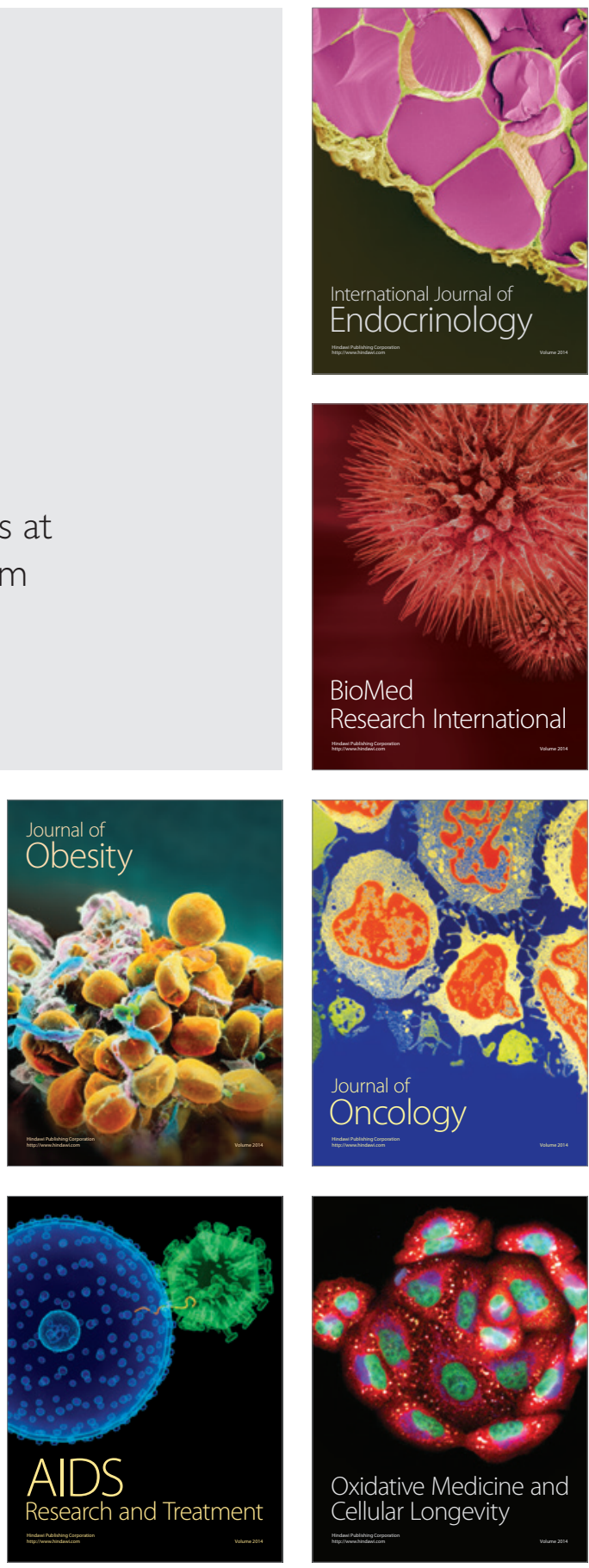